\title{
VISIÓN DEL ALUMNADO UNIVERSITARIO SOBRE EL FOMENTO DEL INGLÉS EN LA EDUCACIÓN SUPERIOR
}

\author{
Martina Soledad Ramírez-Orduña \\ Departamento de Educación, Universidad de Extremadura \\ mramord@unex.es \\ Coral Núñez-Barranco Fernández \\ Departamento de Educación, Universidad de Extremadura \\ coralnf@unex.es \\ Susana Sánchez Herrera \\ Departamento de Psicología, Universidad de Extremadura \\ ssanchez@unex.es \\ María José Rabazo Méndez \\ Departamento de Psicología, Universidad de Extremadura \\ mjrabazo@unex.es
}

Recepción Artículo: 16 mayo 2021

Admisión Evaluación: 16 mayo 2021

Informe Evaluador 1: 27 mayo 2021

Informe Evaluador 2: 02 junio 2021

Aprobación Publicación: 03 junio 2021

\section{RESUMEN}

El fenómeno de la internacionalización ha supuesto en la Educación Superior la adaptación de los planes de estudio al plan Bolonia con el fin de facilitar y fomentar la movilidad académica y laboral de los estudiantes, y mejorar así su competencia idiomática (Fernández et al., 2009; Gallardo et al., 2010). Asimismo, el manejo de un idioma extranjero se ha convertido en la herramienta fundamental en el actual mercado laboral (Luna et al., 2011). Es por eso que las universidades españolas llevan años ofertando programas y ayudas para la movilidad internacional, e incluso han implantado algunos grados en inglés (Sanjosé et al., 2011). El interés del alumnado por participar en programas de movilidad asciende anualmente, habiéndose alcanzado un total de 42.002 participantes durante el curso académico 2017-18 (Ministerio de Ciencia, Innovación y Universidades, 2019). En esta línea, el objetivo de la presente investigación es averiguar la opinión del alumnado universitario acerca del fomento del inglés por parte de la Universidad; para ello han participado un total de 498 sujetos que cursan las titulaciones de Grado y Máster de la Facultad de Educación de Badajoz (Universidad de Extremadura). La metodología empleada ha sido cuantitativa, y se ha realizado una análisis descriptivo e inferencial de los datos. El instrumento empleado para la recogida de datos ha sido un cuestionario de elaboración propia. Entre los resultados obtenidos destaca que el $82,9 \%$ de los sujetos considera que desde la Universidad no se fomenta lo 


\section{VISIÓN DEL ALUMNADO UNIVERSITARIO SOBRE EL FOMENTO DEL INGLÉS EN LA EDUCACIÓN SUPERIOR}

suficiente el aprendizaje del idioma inglés, y 72,9\% opina que la Universidad debería intensificar sus estudios de este idioma.

Palabras clave: bilingüismo; educación superior; inglés; competencia lingüística

\section{ABSTRACT}

University students' view of english language development in higher education. The phenomenon of internationalization in Higher Education has led to the adaptation of curricula to the Bologna Process in order to facilitate and promote students' academic and labor mobility, and thus improve their language proficiency (Fernández et al., 2009; Gallardo et al., 2010). Likewise, foreign language proficiency has become an essential tool in the current job market (Luna et al., 2011). That is why Spanish universities have been offering programs and grants for international mobility for years, and have even implemented some degrees in English (Sanjosé et al., 2011). Students' interest in participating in mobility programs increases annually, having reached a total of 42,002 participants during the 2017-18 academic year (Ministerio de Ciencia, Innovación y Universidades, 2019). In this line, this research aims to find out the opinion of university students about the promotion of English by the University; for this purpose, a total of 498 subjects who are studying Bachelor's and Master's degrees at the Faculty of Education of Badajoz (University of Extremadura) participated in the study. A quantitative methodology was used, and a descriptive and inferential analysis of the data was performed. The data was collected using a questionnaire of our own elaboration. Among the results obtained it is worth noting that $82.9 \%$ of the subjects consider that the University does not promote language acquisition in the English language sufficiently and $72.9 \%$ believe that the University should intensify its studies of this language.

Keywords: bilingualism; higher education; English; linguistic proficiency; language competence

\section{INTRODUCCIÓN}

En los últimos años el fenómeno de la internacionalización ha supuesto en la Educación Superior un gran esfuerzo de adaptación de los planes de estudio al plan Bolonia con el fin de facilitar y fomentar la movilidad académica y laboral de los estudiantes, y mejorar así su competencia idiomática (Fernández et al., 2009; Gallardo et al., 2010). A lo largo de este proceso de internacionalización se han visto influidos directamente varios aspectos fundamentales, siendo los más relevantes la movilidad académica, el papel del inglés como lingua franca y el fomento del multilingüismo (Lasagabaster, 2012). Numerosos estudios (Figueredo y Ortiz, 2017; Hinojosa y López, 2018; Segura-Robles y Parra-Gonzále, 2019), ponen de manifiesto que la interculturalidad se configura como un valor cada vez más importante en la formación inicial del profesorado (Pareja de Vicente et al., 2020).

Debemos pues, ser conscientes de la gran importancia que tiene la educación bilingüe actualmente y tratarla como lo que realmente es: una necesidad para los alumnos (López y Hernández, 2014). Por eso, Gallardo et al., (2010) destacan la necesidad de una competencia multilingüe para poder vivir en la sociedad del siglo XXI, la cual está constantemente cambiando y adaptándose a los retos provocados por la globalización. En este contexto, la necesidad de entender a otros y de ser entendido, obliga a las personas a adentrarse en el dominio de otra lengua (Gallardo et al., 2010); y la necesidad de tener un código común para poder entendernos y relacionarnos, ha hecho del inglés el medio más común para comunicarnos, convirtiéndolo en la lingua franca (López, 2012).

Actualmente, el EEES cuenta con 49 países, de los cuales 47 participan en el Proceso de Bolonia, por lo que se puede considerar la mayor comunidad universitaria a escala mundial, y se organiza conforme a los principios de calidad, movilidad, diversidad y competitividad. Además, persigue dos objetivos estratégicos: incrementar el empleo en la UE y conseguir que estudiantes y profesores de otras partes del mundo se sientan atraídos por el sistema Europeo de Formación Superior (Espacio Europeo Educación Superior, 2015)

Así pues, Salaburu et al., (2011) sostienen que estos hechos han sido los responsables de que las Universidades hayan cambiado profundamente para alcanzar dos objetivos bien marcados. Por un lado, dotar al alumno de las herramientas necesarias para la integración en el ámbito laboral, de forma que pueda dar respuesta 
a las exigencias y demandas de la sociedad actual. Y por otro lado, que los países organicen su educación terciaria de acuerdo con unos principios comunes y generales acordados a nivel europeo.

El Consejo de Europa (2001), y el El Marco Común Europeo de Referencia de las Lenguas (MCERL), proporcionan una base común a toda Europa, en la que se describen los conocimientos y destrezas que tienen que adquirir los estudiantes con el fin de utilizar eficazmente una determinada lengua para comunicarse.

Las competencias lingüísticas generales, específicas y transversales están definidas en la Universidad de Extremadura (UEX) con certificación de la ANECA para los estudios de Grado de Educación Infantil y Primaria, así como los Másteres en los que se centra este trabajo. En estos estudios se exige un nivel B1 (según niveles establecidos en el MCERL) en competencia oral y escrita, y en el caso de los Másteres conocer y fomentar la metodología AICLE en sus aulas.

En este contexto, el manejo de un idioma extranjero se ha convertido en la herramienta fundamental tanto el actual mercado laboral como en el ámbito educativo (Luna et al., 2011), por eso las universidades españolas Ilevan años ofertando programas y ayudas para la movilidad internacional, e incluso han implantado algunos grados en inglés (Sanjosé et al., 2011).

Respecto a la organización de los programas de la Universidad de Extremadura son gestionados por el Vicerrectorado de Relaciones Institucionales e Internacionalización, y se rigen por la Normativa Reguladora de los Programas de Movilidad de la UEx, donde se recogen los derechos y obligaciones que deberán cumplir los alumnos que participan en los programas de movilidad. Entre los programas que oferta la UEx enfocados a la movilidad internacional de sus estudiantes destacan Erasmust, Erasmus Mundus, becas internacionales Americampus, becas Iberoamérica Santander Universidades, programa de prácticas en el Instituto Tecnológico, etc.

El alumnado, consciente de la necesidad de mejorar su competencia lingüística, ha aumentado su participación en este tipo de programas de movilidad; produciéndose un ascenso anual y habiéndose alcanzado más de 42.002 participantes durante el curso académico 2017-18 (Ministerio de Ciencia, Innovación y Universidades, 2019).

\section{OBJETIVOS DE LA INVESTIGACIÓN}

El objetivo general de este estudio ha sido realizar un análisis sobre la satisfacción del alumnado universitario respecto a la enseñanza del inglés por parte de la Universidad.

Más concretamente nos hemos planteado un total de seis objetivos específicos (OE) que nos guiarán en nuestra investigación:

-0E1: Averiguar si la titulación que cursa el alumnado influye en el contacto que estos tienen con profesores nativos durante su etapa universitaria.

- OE2: Descubrir si el nivel de exigencia en materia lingüística del alumnado hacia la Universidad varía según si estos poseen un certificado oficial de LE, o no.

- OE3: Conocer el grado de satisfacción del alumnado en cuanto al fomento del inglés por parte de la Universidad en función de si poseen, o no, certificado oficial de LE.

-0E4: Saber si la titulación que cursan los alumnos influye en su opinión respecto a la necesidad de intensificar el estudio del idioma inglés por parte de la Universidad.

- OE5: Descubrir si los alumnos que poseen un certificado oficial de LE consideran, en mayor medida que los que no lo poseen, que es necesario intensificar el estudio del idioma inglés por parte de la Universidad.

- OE6: Averiguar si los alumnos que poseen un certificado oficial de LE son, en mayor medida que los que no lo poseen, partidarios de que se establezca un modelo bilingüe en la universidad.

\section{MUESTRA}

Los participantes en esta investigacion han ascendido a 498 sujetos pertenecientes a diversos grupos de las titulaciones de Grado y Máster (Tabla 1) de de la Facultad de Educacion del campus universitario de Badajoz (UEX). 


\section{VISIÓN DEL ALUMNADO UNIVERSITARIO SOBRE EL FOMENTO DEL INGLÉS EN LA EDUCACIÓN SUPERIOR}

La selección de la muestra se realizó mediante muestreo no probabilistico por conveniencia, participando aquellos alumnos cuyos profesores accedieron a colaborar con el estudio.

La edad de los sujetos estuvo comprendida entre los 18 y los 49 años ( $M=22,52 ; D T=4,013)$; el 83\% de la muestra ( $n=413$ sujetos) se encuentra cursando estudios de Grado y el 17\% restante ( $n=85$ sujetos) estudios de Máster.

Tabla 1. Distribución de la muestra según la titulación que cursa

\begin{tabular}{llrr}
\hline & & \multicolumn{2}{c}{ Total sujetos } \\
\cline { 3 - 3 } & & $\mathbf{n}$ & $\%$ \\
\hline $\begin{array}{l}\text { Titulaciones de } \\
\text { Grado }\end{array}$ & Educación Infantil & 193 & 38,8 \\
& Educación Primaria & 220 & 44,2 \\
\cline { 2 - 3 } & Máster Universitario de Investigación en Ciencias Sociales y Jurídicas & 37 & 7,4 \\
Titulaciones de & Máster & Méster Universitario en Investigación en la Enseñanza y el Aprendizaje & 13 \\
& Máster Universitario en Enseñanza Bilingüe para la Educación Primaria & 2,6 \\
& y Secundaria & 9 & 1,8 \\
& Máster Universitario en Formación del Profesorado de Educación & 26 & 5,2 \\
\hline
\end{tabular}

$n=$ Frecuencia de sujetos $\quad \%=$ Porcentaje de sujetos

\section{METODOLOGÍA E INSTRUMENTOS UTILIZADOS}

La metodología empleada responde a un modelo cuantitativo descriptivo, no experimental y transversal y el instrumento empleado se trata de un cuestionario de elaboración propia validado por un panel de expertos de la UEx. El cuestionario cuenta con 25 preguntas configuradas como variables categóricas dicotómicas (si/no), politómicas empleando una escala Likert y preguntas abiertas, de las cuales hemos seleccionado 11 ítems que recogen la información necesaria para realizar el estudio.

Una vez recopilados todos los cuestionarios se procedió a realizar un un análisis estadístico empleando el paquete SPSS (Statistical Product and Service Solutions). Se desarrolló primero un analisis descriptivo de las variables, y posteriormente un analisis inferencial utilizando pruebas no paramétricas.

\section{RESULTADOS ALCANZADOS}

Los resultados obtenidos del analisis descriptivo e inferencial se muestran en funcion de los objetivos específicos planteados.

\section{Objetivo Específico 1.}

Como se observa en la Tabla 2, más de la mitad del alumnado de todas las titulaciones, excepto del Máster Universitario en Enseñanza Bilingüe, no han tenido profesores nativos durante su etapa universitaria. Además encontramos una gran diferencia entre los alumnos que cursan el Grado de Ed. Infantil, donde hallamos que la gran mayoría $(85,5 \%)$ no han tenido contacto con profesores nativos de inglés durante su paso por la universidad. 
Tabla2. Distribución de la muestra según si han tenido profesores nativos en la universidad

\begin{tabular}{|c|c|c|c|c|c|}
\hline & & \multicolumn{2}{|c|}{ Sí } & \multicolumn{2}{|c|}{ No } \\
\hline & & n & $\%$ & $\mathbf{n}$ & $\%$ \\
\hline \multirow{6}{*}{ Titulación } & Educación Infantil & 28 & 14,5 & 165 & 85,5 \\
\hline & Educación Primaria & 86 & 39,1 & 134 & 60,9 \\
\hline & Máster Universitario de Investigación en Ciencias Sociales y Jurídicas & 9 & 24,3 & 28 & 75,7 \\
\hline & $\begin{array}{l}\text { Máster Universitario en Investigación en la Enseñanza y el } \\
\text { Aprendizaje de las Ciencias Experimentales, Sociales y Matemáticas }\end{array}$ & 6 & 46,2 & 7 & 53,8 \\
\hline & $\begin{array}{l}\text { Máster Universitario en Enseñanza Bilingüe para la Educación } \\
\text { Primaria y Secundaria }\end{array}$ & 8 & 88,9 & 1 & 11,1 \\
\hline & $\begin{array}{l}\text { Máster Universitario en Formación del Profesorado de Educación } \\
\text { Secundaria }\end{array}$ & 3 & 11,5 & 23 & 88,5 \\
\hline
\end{tabular}

Además, mediante la prueba Chi Cuadrado (Tabla 3) comprobamos que existen diferencias significativas ( $p=$ ,000) en el porcentaje de alumnos que han sido expuestos a docencia impartida por profesores nativos de inglés según la titulación que cursa, por lo que confirmamos que la titulación que el alumnado cursa es un factor determinante en el contacto que este tiene con profesores nativos durante su etapa universitaria.

Tabla3. Prueba Chi-Cuadrado en la relación al contacto del alumnado con profesores nativos según la titulación que cursa

\begin{tabular}{llrl}
\hline & & \multicolumn{1}{c}{ Valor } & \multicolumn{1}{c}{ Sig. } \\
\cline { 3 - 4 } Prueba Chi-cuadrado de Pearson & & 53,137 &, $000^{* *}$ \\
\hline Sig.= Significación estadistica & ${ }^{* *} p \leq, 010$ & ${ }^{*} p \leq, 050$ &
\end{tabular}

\section{Objetivo Específico 2.}

En este caso la muestra fue clasificada dependiendo de si poseía, o no, una titulación oficial de LE. Como refleja la Tabla 4, la mayoría de los sujetos que sí poseen este certificado de LE $(83,5 \%)$ considera que la Universidad debería exigir una acreditación de lengua inglesa al alumnado antes de que este finalice sus estudios universitarios. Sin embargo, este porcentaje desciende al $66,1 \%$ cuando se trata de sujetos que no poseen una acreditación de lengua inglesa.

Tabla 4. Distribución de la muestra, con y sin certiicación oficial de LE, según la necesidad de acreditar idioma antes de finalizar estudios universitarios

\begin{tabular}{|c|c|c|c|c|c|}
\hline & & \multicolumn{2}{|c|}{$\begin{array}{c}\text { Poseen titulación } \\
\text { oficial = SI }\end{array}$} & \multicolumn{2}{|c|}{$\begin{array}{c}\text { Poseen titulación } \\
\text { oficial = NO }\end{array}$} \\
\hline & & $\mathbf{n}$ & $\%$ & $\mathbf{n}$ & $\%$ \\
\hline ¿Cree que la Universidad debería exigir una & Sí & 76 & 83,5 & 269 & 66,1 \\
\hline $\begin{array}{l}\text { acreditación oficial de lengua inglesa antes de } \\
\text { finalizar los estudios universitarios? }\end{array}$ & No & 15 & 16,5 & 136 & 33,4 \\
\hline
\end{tabular}




\section{VISIÓN DEL ALUMNADO UNIVERSITARIO SOBRE EL FOMENTO DEL INGLÉS EN LA EDUCACIÓN SUPERIOR}

Tabla 5. Prueba CHI-Cuadrado en la relación a la necesidad de acreditar idioma antes de finalizar estudios universitarios según si el alumnado posee, o no, certificación oficial de LE

\begin{tabular}{|c|c|c|}
\hline & Valor & Sig. \\
\hline Prueba Chi-cuadrado de Pearson & 10,257 &, $001^{* *}$ \\
\hline
\end{tabular}

Con el objetivo de comprobar si estas diferencias son estadísticamente significativas realizamos de nuevo la prueba no paramétrica Chi Cuadrado (Tabla 5).

En base a los datos arrojados por la anterior tabla, las diferencias halladas previamente resultan ser estadísticamente significativas $(p=, 001)$, por lo que confirmamos que los alumnos que poseen una certificación oficial de LE consideran, en mayor medida que los que no poseen dicha certificación, que la universidad debería exigir que todos los alumnos logren acreditarse de lengua inglesa antes de terminar los estudios universitarios.

\section{Objetivo Específico 3.}

Mediante este objetivo queremos saber la opinión de los alumnos respecto al fomento del idioma inglés por parte de la Universidad (Tabla 6). Para ello clasificamos la muestra según si posee certificación oficial de LE 0 no la posee.

Tabla 6. Distribución de la muestra, con y sin certificación oficial de LE, sobre el fomento del qprendizaje del inglés por parte de la Universidad

\begin{tabular}{|c|c|c|c|c|c|}
\hline & & \multicolumn{2}{|c|}{$\begin{array}{l}\text { Poseen titulación } \\
\text { oficial = SI }\end{array}$} & \multicolumn{2}{|c|}{$\begin{array}{l}\text { Poseen titulación } \\
\text { oficial = NO }\end{array}$} \\
\hline & & $\mathbf{n}$ & $\%$ & $\mathbf{n}$ & $\%$ \\
\hline \multirow{3}{*}{$\begin{array}{l}\text { ¿Piensa que desde la Universidad se } \\
\text { fomenta el aprendizaje del inglés? }\end{array}$} & Sí & 7 & 7,7 & 78 & 19,2 \\
\hline & No lo suficiente & 59 & 64,8 & 249 & 61,2 \\
\hline & No & 25 & 27,5 & 80 & 19,7 \\
\hline
\end{tabular}

Llama la atención que más de la mitad de los sujetos de ambos grupos (64,8\% en sujetos con titulación oficial y $61,2 \%$ en sujetos sin titulación oficial de LE) consideran que la universidad no fomenta lo suficiente el aprendizaje del idioma inglés.

Tabla 7. Prueba U Mann-Whitney en relación al fomento del aprendizaje del idioma inglés por parte de la Universidad según si el alumnado posee, o no, certificación oficial de LE

\begin{tabular}{llll}
\hline & & Valor & \multicolumn{1}{c}{ Sig. } \\
\cline { 3 - 4 } Prueba U de Mann-Whitney & & 15641,500 &, $007^{* *}$ \\
\hline Sig. = Significación estadistica & ${ }^{* *} p \leq, 010$ & ${ }^{*} p \leq, 050$ &
\end{tabular}

La significación hallada mediante la prueba U de Mann-Whitney ( $p=, 007)$, y reflejada en esta Tabla 7, nos indica que existen diferencias estadísticamente significativas en la opinión del alumnado respecto al fomento del idioma inglés por parte de la Universidad, siendo los sujetos que poseen una titulación oficial de LE los que consideran, en mayor medida que los que no poseen titulación, que la Universidad no fomenta el aprendizaje de este idioma lo suficiente.

\section{Objetivo Específico 4.}

Otro de los objetivos planteados ha sido conocer si la opinión del alumnado en cuanto a la necesidad de intensificar el estudio del idioma inglés por parte de la Universidad difiere según la titulación que éste cursa. 
Tabla 8. Distibución de la muestra según si creen que la Universidad debe intensificar el estudio del idioma inglés

\begin{tabular}{|c|c|c|c|c|c|}
\hline & & \multicolumn{2}{|c|}{ Sí } & \multicolumn{2}{|c|}{ No } \\
\hline & & n & $\%$ & $\mathbf{n}$ & $\%$ \\
\hline \multirow{6}{*}{ Titulación } & Educación Infantil & 133 & 69,3 & 59 & 30,7 \\
\hline & Educación Primaria & 157 & 71,7 & 62 & 28,3 \\
\hline & Máster Universitario de Investigación en Ciencias Sociales y Jurídicas & 28 & 75,7 & 9 & 24,3 \\
\hline & $\begin{array}{l}\text { Máster Universitario en Investigación en la Enseñanza y el } \\
\text { Aprendizaje de las Ciencias Experimentales, Sociales y Matemáticas }\end{array}$ & 12 & 92,3 & 1 & 7,7 \\
\hline & $\begin{array}{l}\text { Máster Universitario en Enseñanza Bilingüe para la Educación } \\
\text { Primaria y Secundaria }\end{array}$ & 9 & 100 & 0 & 0,0 \\
\hline & $\begin{array}{l}\text { Máster Universitario en Formación del Profesorado de Educación } \\
\text { Secundaria }\end{array}$ & 24 & 92,3 & 2 & 7,7 \\
\hline
\end{tabular}

$n=$ Frecuencia de sujetos $\quad \%=$ Porcentaje de sujetos

El análisis descriptivo (Tabla 8) muestra cómo la gran mayoría del alumnado considera que la Universidad debería intensificar el idioma inglés. Destaca especialmente el alumnado del Máster en Enseñanza Bilingüe con el 100\% de los sujetos considerando que es necesario realizar este cambio.

El análisis inferencial (Tabla 9), muestra que existen diferencias estadísticamente significativas $(p=, 029)$ entre la opinión del alumnado respecto a este tema dependiendo de la titulación que estos cursan.

\section{Objetivo Específico 5.}

El objetivo planteado se centra en descubrir si la opinión que el alumnado que posee respecto a la necesidad de intensificar el estudio del idioma inglés por parte de la Universidad varía según si estos poseen certificación oficial de LE.

\begin{tabular}{|c|c|c|}
\hline & Valor & Sig. \\
\hline Prueba Chi-cuadrado de Pearson & 12,430 &, $029^{*}$ \\
\hline
\end{tabular}

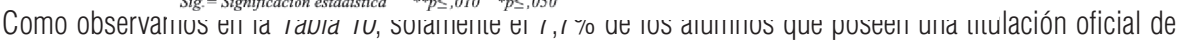
LE consideran que no es necesario intensificar el estudio del idioma inglés por parte de la Universidad; de los alumnos que no poseen una titulación oficial de LE, el 31\% comparte esta misma opinión.

Para comprobar si estas diferencias resultan ser estadísticamente significativas, realizamos la prueba Chi Cuadrado (Tabla 11), la cual nos reportó un nivel de significación de $p=, 000$.

En base a estos resultados podemos confirmar que la opinión del alumnado respecto a la necesidad de intensificar el estudio del idioma inglés por parte de la Universidad varía según si estos poseen, o no, titulación de LE, siendo los que sí poseen dicha titulación más exigentes que los que no la poseen.

\section{Objetivo Específico 6.}

Finalmente quisimos conocer si la muestra se mostraba a favor o en contra del establecimiento de un modelo bilingüe en la Universidad; para ello volvimos a clasificar a los sujetos según si poseían, o no, certificado de LE.

El análisis descriptivo desarrollado en la Tabla 12 muestra que el 69,2\% de alumnos que sí poseen una certificación de LE están a favor de que se implante un modelo bilingüe en la universidad; este porcentaje desciende al 53,8\% cuando se trata de sujetos que no poseen certificación de LE.

En cuanto al análisis inferencial (Tabla 13), nos indica que las diferencias halladas anteriormente son estadísticamente significativas ( $p=, 007$ ) por lo que confirmamos que los alumnos que poseen un certificado 


\section{VISIÓN DEL ALUMNADO UNIVERSITARIO SOBRE EL FOMENTO DEL INGLÉS EN LA EDUCACIÓN SUPERIOR}

oficial de LE son, en mayor medida que los que no lo poseen, partidarios de que se establezca un modelo bilingüe en la universidad.

\section{DISCUSIÓN}

Los datos obtenidos en este estudio descriptivo nos proporcionan información interesante y significativa. En general, predomina que únicamente el 18,3\% del alumnado posee una certificación oficial de LE, de los cuales un $16,3 \%$ es de inglés. Es decir, este alumnado, al margen de sus estudios universitarios, ha querido obtener una certificación oficial a través de exámenes de la Escuela Oficial de Idiomas (EOI), University of Cambridge, Instituto de Lenguas Modernas, u otro organismo examinador; lo cual nos muestra un interés y una motivación por parte del alumnado hacia este idioma. No obstante, este dato es alarmante, ya que se trata de un porcentaje muy bajo considerando que nos encontramos ante futuros maestros. Sin embargo, estos datos se aproximan a los hallados en el estudio realizado por López et al., (2013), donde tan sólo un 13,6\% de la muestra seleccionada para su investigación (alumnos del Grado de Enfermería de la Universidad de Cádiz) poseía una acreditación oficial de lengua inglesa.

Comprobamos también en nuestra investigación que, de los alumnos que poseen certificación oficial de inglés, el 83,5\% ha alcanzado el nivel B1 o superior. Estos datos son positivos si los comparamos con los obtenidos por Gómez et al., (2014) en el estudio realizado en la Facultad de Magisterio de Valencia a estudiantes de $2^{0}$ año de carrera; ya que en esta investigación más de la mitad de la muestra $(51,4 \%)$ se situaba en los niveles A1-A2 (nivel principiante) y solo un 48,5\% consiguió acreditarse de un nivel B1 o superior (niveles establecidos según los estándares europeos - MCERL).

Otro aspecto positivo es que más de la mitad de la muestra recogida en este estudio (53\%) tiene pensado examinarse próximamente para poder obtener dicha titulación, y de ellos, un 99,2\%, lo harán de idioma inglés; lo que refleja que son conscientes de la importancia que tiene este idioma en la sociedad actual (Berenguer, et al., 2018; Eurydice, 2017; Martín del Pozo, 2013; Raigón, 2015; Raigón y Larrea, 2018).

Un análisis detallado sobre la satisfacción del alumnado respecto a la enseñanza del inglés por parte de la Universidad muestra que el 73,2\% considera que se debería intensificar la enseñanza-aprendizaje del idioma inglés, ya que, de acuerdo con el 82,9\% de los sujetos, la Universidad no fomenta lo suficiente 0 , simplemente, no fomenta el aprendizaje de la lengua inglesa. Estos resultados se aproximan a los encontrados en López et al., (2013), donde el 91,8\% de los participantes consideraba que "la Universidad ofrece recursos insuficientes para manejar el inglés en el nivel exigido por la normativa" (p.2).

Hemos comprobado que el $69,93 \%$ de los sujetos que conforman la muestra está a favor de que la Universidad exija una acreditación oficial de lengua inglesa antes de que los alumnos acaben sus estudios, dato que discrepa del hallado por Lopez et al., (2013) en su estudio, en el cual la mayoría de la muestra (Grado de enfermería) consideraba inadecuado que se exigiera tal acreditación. Además, el 56,6\% de la muestra está a favor de establecer un modelo de enseñanza bilingüe en la Universidad ya que únicamente un 28,1\% afirma haber tenido profesores nativos durante su etapa en la Universidad.

En cuanto a los objetivos específicos, hemos comprobado que los alumnos que poseen titulación oficial de LE consideran, en mayor medida que los que no poseen dicha titulación, que la Universidad debería exigir una acreditación de lengua inglesa al alumnado antes de que éste finalice sus estudios universitarios. Estos alumnos también destacan significativamente sobre los que no poseen titulación oficial de LE, al considerar que la Universidad no fomenta lo suficiente el estudio del idioma inglés; que se debería intensificar el estudio del mismo; y al posicionarse a favor de implantar programas de educación bilingües.

El análisis realizado por ramas de conocimiento o titulaciones ha reportado la existencia de diferencias estadísticamente significativas en la exposición y contacto del alumnado a profesores nativos durante tu etapa universitaria, así como en la opinión respecto a la necesidad de intensificar los estudios de inglés. Estos resultados concuerdan con Amengual-Pizarro (2018) y Gil-Galván y Martín-Espinosa (2021) donde se observó 
también que el el alumnado presentaba actitudes dispares dependiendo de la titulación que cursa. En este primer estudio el alumnado de primaria manifiestan una actitud más favorable que el de infantil; posiblemente debido a que "para el alumnado de Educación Primaria una posible salida profesional es la de docente especializado en inglés, mientras que el alumnado de Educación Infantil no puede acceder a ese perfil docente" (Aranberri, 2021, p.33).

\section{CONCLUSIONES}

Tras el análisis realizado, podemos concluir que el inglés sigue siendo el principal idioma elegido por el estudiantado. Tanto es así, que más de la mitad de los sujetos están a favor de que en la Universidad se establezca un sistema bilingüe.

En coherencia con lo anterior, es interesante destacar que son los propios alumnos los que reflejan que la Universidad debería intensificar sus estudios del idioma inglés, así como exigir una acreditación oficial de lengua inglesa antes de que finalicen sus estudios.

En relación a los niveles que están estudiando para acreditarse en un idioma, los datos constatan que la mayoría estudian para el nivel B1, datos en consonancia con otras investigaciones donde se vislumbra cómo dentro de Ciencias de la Educación existen Grados con motivaciones muy dispares a la hora de aprender idiomas (Gil-Galván y Martín-Espinosa, 2021).

Las diferencias significativas halladas en cuanto al nivel de acreditación del alumando en nuestro estudio podrían estar estrechamente relacionada con diversos estudios que han demostrado la existencia de una correlación positiva entre niveles de exigencia y nivel de competencia lingüística (Alejo y Piquer-Piriz, 2016; De Castro, 2014).

\section{REFERENCIAS BIBLIOGRÁFICAS}

Alejo, R., y Piquer-Piriz, A. (2016). Urban vs. rural CLIL: An analysis of input-related variables, motivation and language attainment. Language, Culture and Curriculum. DOI: http://dx.doi.org/10.1080/07908318.2016.1154068

Aranberri, N., Bereziartua, G., y Boillos, M. M. (2021). La motivación del futuro profesorado bilingüe hacia el aprendizaje del inglés. Profesorado. Revista deCurrículum y Formación de Profesorado, 25(1), 319-337. D0I:1030827/profesorado.v25i3.8658

Berenguer, R., Mateo-Guillén, C., Ramos-Fernández, E. V., Montilla, F., y Morallón, E. (2018). Estudio de los conocimientos de inglés técnico/específico de química en los Grados de Química y Geología de la Universidad de Alicante. En R. Roig-Vila (Ed.), El compromiso académico y social a través de la investigación e innovación educativas en la Enseñanza Superior (49-58). Barcelona: Octaedro.

Consejo de Europa (2001). Marco común europeo de referencia para las lenguas: Aprendizaje, enseñanza, evaluación. Madrid: Ministerio de Educación, Cultura y Deporte, Instituto Cervantes, Anaya. Recuperado de http://cvc.cervantes.es/ensenanza/ biblioteca_ele/marco/cvc_mer.pdf

De Castro, R. M. (2014). La ensenanza bilingue en Madrid. Terbiya, 43(2), 197-209.

Eurydice. (2017). Key Data on Teaching Languages at School in Europe 2017. Brussels: Eurydice European Unit. https://eacea. ec.europa.eu/national-policies/eurydice/sites/eurydice/files/es_kdl_infog_2017_1.pdf

Fernandez, S., Perez, C., y Vaquero, A. (2009). Movilidad internacional de la Universidad Espanola: Analisis regional e institucional del programa Socrates-Erasmus. Revista de Estudios Regionales, 85, 134-172.

Figueredo, V., y Ortiz, L. (2017). Formación inicial del profesorado para la inclusión de la diversidad cultural. Revista Digital de Investigación en Docencia Universitaria, 11(1), 38-61.

Gallardo, D., Sanchez, M. I., y Corchuelo, M. B. (2010). Competencias transversales en el EEES: La opinion del estudiante sobre la importancia y el dominio de una lengua extranjera. Revista de Educacion en Contabilidad, Finanzas y Administracion de Empresas, 1(1), 79-99. 


\section{VISIÓN DEL ALUMNADO UNIVERSITARIO SOBRE EL FOMENTO DEL INGLÉS EN LA EDUCACIÓN SUPERIOR}

Gil-Galván, R., y Martín-Espinosa, I. (2021). ¿Cómo valoran los estudiantes universitarios de Educación su dominio de competencias lingüísticas en lengua extranjera? Revista Complutense de Educación, 32(2), 237247.

Hinojosa, E. F., y López, M. (2018). Interculturality and Teacher Education. A Study From Pre-service Teachers' Perspective. Australian Journal of Teacher Education, 43(3), 5.

Lasagabaster, D. (2012). El papel del inglés en el fomento del multilingüismo en la Universidad. Estudios de Lingüística Inglesa Aplicada, ELIA, 12, 13-44.

López, A. (2012). Factores que influyen en la motivación del aprendiente en el aprendizaje del español o del inglés como lenguas extranjeras en una universidad de Taiwán, y en otra de Asunción, Paraguay. Revista Internacional de Investigación en Ciencias Sociales, 8(2), 207-220.

Lopez, C., Abellan, M. J., Almenara, J., Lorenzo, J. R., Castro, C., Carnicer, I., Moreno, L., Santi, M. J., Picardo, J. M. (2013). Importancia del ingles en la formacion de Grado de Enfermeria bajo la perspectiva de los estudiantes. Proyectos de innovacion y mejora docente.

López, E., y Hernández, T. (2014). Enfoque y metodología en las aulas bilingües. Formación continuada Logos.

Luna, M., Barradas, M. E. y Balderrama, J. A. (2011). Depresion en estudiantes con rezago en Ingles I y II. Universidad Veracruzana. Revista Iberoamericana para la Investigacion y el Desarrollo Educativo, 2(3), 177188.

Martín del Pozo, M. A. (2013). Formación del profesorado universitario para la docencia en inglés. Revista de Docencia Universitaria, 11(3), 197-218. https://polipapers.upv.es/index.php/REDU/article/view/5526

Ministerio de Ciencia, Innovación y Universidades (2019). Datos y cifras del Sistema.

Pareja de Vicente, D., Leiva, J. J., y Matas, A. (2020). Percepciones sobre diversidad cultural y comunicación intercultural de futuros maestros. Revista Electrónica Interuniversitaria de Formación del Profesorado, 23(1), 75-87. DOI: https://doi.org/10.6018/reifop.403331

Pedraza-Rodríguez, J. A. (2021). Consolidación como buena práctica docente: La metodología AICLE en el desarrollo de la docencia en el grado de turismo de la Universidad de Córdoba. Revista de Innovación y Buenas Prácticas Docentes, 10(1), 15-28.

Raigón, A. R. (2015). The impact of introducing foreign language requirements on the Spanish higher education system: The case of Córdoba University. CercleS, 5(1), 265-279. https://www.degruyter.com/view/j/cercles.2015.5.issue-1/cercles-2015-0013/ cercles-2015-0013.xml

Raigón, A. R. y Larrea, A. (2018). Estudio comparativo de las acreditaciones lingüísticas usadas por el alumnado de Grado de la Universidad de Córdoba. E-Aesla, 4, 190-203. https://cvc.cervantes.es/lengua/eaesla/pdf/04/19.pdf

Salaburu, P., Haug, G., y Mora, J. G. (2011). Espana y el proceso de Bolonia. Un encuentro imprescindible. Madrid: Academia Europea de Ciencia y Artes.

Sanjose, V., Solaz, J. J., y Gomez, A. (2011). Control de la comprension durante la lectura de textos de ciencias en ingles. ¿Estan preparados los estudiantes universitarios espanoles para integrarse en el Espacio Europeo de Educacion Superior? Didactica de las Ciencias Experimentales y Sociales, 25, 149-163.

Segura Robles, A., y Parra González, M. E. (2019). Analysis of Teachers' Intercultural Sensitivity Levels in Multicultural Contexts. Sustainability, 11(11), 21-37. 